\title{
Acute Pergolide Exposure Stiffens Engineered Valve Interstitial Cell Tissues and Reduces Contractility In Vitro
}

Andrew K. Capulli, Luke A. MacQueen, Blakely B. O'Connor, Stephanie Dauth, Kevin Kit Parker*

\author{
*Corresponding Author: \\ Kevin Kit Parker, Ph.D. \\ Disease Biophysics Group \\ Wyss Institute for Biologically Inspired Engineering \\ John A. Paulson School of Engineering and Applied Sciences \\ Harvard University \\ 29 Oxford St, Pierce Hall 321 \\ Cambridge MA 02138 \\ E-mail: kkparker@seas.harvard.edu
}

\section{Affiliation}

Disease Biophysics Group. Wyss Institute for Biologically Inspired Engineering, John A. Paulson School of Engineering and Applied Sciences, Harvard University, 29 Oxford St, Pierce Hall 321, Cambridge, MA 02138, USA. 


\begin{abstract}
Medications based on ergoline-derived dopamine and serotonin agonists are associated with offtarget toxicities that include valvular heart disease (VHD). Reports of drug-induced VHD resulted in the withdrawal of appetite suppressants containing fenfluramine and phentermine from the U.S. market in 1997 and pergolide, a Parkinson's disease medication, in 2007. Recent evidence suggests that serotonin receptor activity affected by these medications modulates cardiac valve interstitial cell activation and subsequent valvular remodeling, which can lead to cardiac valve fibrosis and dysfunction similar to that seen in carcinoid heart disease. Failure to identify these risks prior to market, and continued use of similar drugs, reaffirms the need to improve preclinical evaluation of drug-induced VHD. Here, we present two complimentary assays to measure stiffness and contractile stresses generated by engineered valvular tissues in vitro. As a case study, we measured the effects of acute $(24 \mathrm{hr})$ pergolide exposure to engineered porcine aortic valve interstitial cell (AVIC) tissues. Pergolide exposure led to increased tissue stiffness but it decreased both basal and active contractile tone stresses generated by AVIC tissues. Pergolide exposure also disrupted AVIC tissue organization (i.e., tissue anisotropy), suggesting that the mechanical properties and contractile functionality of these tissues are governed by their ability to maintain their structure. We expect further use of these assays to identify off-target drug effects that alter the phenotypic balance of AVICs, disrupt their ability to maintain mechanical homeostasis, and lead to VHD.
\end{abstract}

KEY WORDS: Valvular Heart Disease, Pergolide, Valve Interstitial Cells, Ergoline, Serotonin Agonist 


\section{Introduction}

A growing number of medications such as those used in the treatment of Parkinson's disease and psychiatric disorders are dopamine/serotonin analogs or are designed to stimulate the production/reuptake of these neurotransmitters in the brain [1,2]. Although both dopamine and serotonin are drug targets for neurological pharmacotherapies, their expression is ubiquitous throughout the body. In particular, they are highly expressed in the gastrointestinal and pulmonary tracts, as well as throughout the cardiovascular system, including the myocardium and cardiac valves [3, 4]. Consequently, chronic use of these drugs can cause significant patient morbidity via off-target, adverse effects such as pulmonary and cardiac fibrosis [5-8]. Ergolinederived medications appear to be especially problematic as a number of anorectics [7] and Parkinson's disease drugs [9] of this class cause fibrosis and gross structural changes to the cardiac valves, similar to the symptoms of carcinoid syndrome [7, 8]. These cases of druginduced valvular heart disease (VHD) are believed to result from excessive, chronic valvular interstitial cell (VIC) activation by serotonin 5HT-2B receptor agonism [10, 11]. Conversely, 5HT-2B antagonism inhibits myofibroblast activation of VICs in vitro, with increasing doses of 5HT-2B antagonist in the presence of transforming growth factor beta-1 (TGF- $\beta 1$ ) leading to progressive decreases in alpha smooth muscle actin ( $\alpha$-SMA) expression after $24 \mathrm{hr}$ [12]. Ergoline-derived medications therefore appear to be potent regulators of VIC activation and valve repair homeostasis but the time-course of action, progression to tissue-level mechanical dysregulation and potential reversibility are understudied. We reasoned that mechanisms by which VIC activation translates to VHD could be studied in vitro using assays based on controlled VIC assembly into functional tissues that recapitulate key properties of natural valves.

VICs are a heterogeneous population of predominantly fibroblast-like cells found in all 
three layers of the valve leaflet extracellular matrix (ECM). VIC populations are rich in mesenchymal stem cells that have robust osteogenic calcification potential [13] and VIC activation from the quiescent fibroblast phenotype to a contractile myofibroblast phenotype (expressing $\alpha$-SMA and SM22 $\alpha$ [14]) confers reparative capacity to healthy valves $[15,16]$ and an ability to generate tissue tone [17]. VICs demonstrate a natural plasticity between these activated and quiescent phenotypes largely in response to their biochemical and mechanical environments. For example, TGF- $\beta$ has been shown to cause dose-dependent activation of VICs and ECM remodeling [18, 19]. Mechanical properties of the valve niche that regulate VIC activation include transvalvular pressures, ECM stiffness, and fluidic shear forces [20-24]. Drugs can dysregulate the biochemical and mechanical homeostasis of VICs and cause chronic, pathological VIC activation that can lead to exaggerated matrix deposition, fibrosis, and calcification [7-10]. The eventual clinical manifestation of pathologically activated VICs presents as late-stage permanent valvular tissue dysfunction [25, 26]. In vitro assays aimed at identifying mechanisms of drug-induced VHD would benefit from tissue-level functional metrics that tie VIC activation to valve performance.

Acute, in vitro detection of pathological VIC differentiation has focused on mitogenic signaling pathways involved in excessive 5HT-2B receptor agonism, that include ERK1/2, Src, PKC, and/or TGF- $\beta 1[10,27-30]$. These provide insight into potential treatment strategies but acute functional changes to valve tissues resulting from dysregulated activation of these pathways remain largely unknown and undiagnosable clinically. We therefore asked whether VIC-based tissue stiffness and tissue tone stress generation were altered by acute exposure to 5HT-2B agonists. To answer this question, we engineered VIC tissues and measured changes in stiffness in response to exposure to $8 \beta$-methylthiomethyl-6-propylergoline (pergolide), a potent 
5HT-2B receptor agonist [31]. We then designed and built an in vitro VIC tissue tone stress assay to determine the effects of pergolide on the tissue's capacity to generate contractile stresses. By exposing engineered VIC tissues to a clinically relevant dose of pergolide, we demonstrate that the characteristic tissue stiffening and disorganization of drug-induced valvulopathy is detectable after acute $24 \mathrm{hr}$ drug exposure in vitro. Using our tissue tone assay, we demonstrate that acute pergolide exposure reduced VIC tissue contractile capacity. Our results indicate that pergolide-induced mechanical abnormalities that lead to clinically dysfunctional heart valve performance can be detected in vitro after acute exposures. These tissue-level, mechanical assays may provide a platform for drug screening and investigation into early stage drug-induced VHD.

\section{Methods}

We developed two distinct in vitro assays to measure VIC tissue stiffness and tone generation in response to acute pergolide exposure. Although fibrotic carcinoid-like valvular pathologies occur primarily in the right heart valves (tricuspid and pulmonary) due to pulmonary clearance of excessive serotonin [32], pergolide causes both left (aortic and mitral) and right (pulmonary and tricuspid) side valve dysfunction [6, 33-35]. Because the aortic valve fibrosa layer is most commonly associated with disease [36, 37], we engineered aortic VIC (AVIC) tissues that recapitulated aortic fibrosa ECM structure (Fig. 1A). For tissue stiffness measurements, we cultured AVICs on thin fibrous scaffolds that recapitulated the stiffness and structural anisotropy of healthy valve fibrosa tissues. Equibiaxial loading was used to measure the directionally dependent tensile elastic modulus of AVIC tissues exposed to pergolide. To measure tissue tone stress regulation, we cultured anisotropic AVIC tissues on thin flexible cantilevers based on muscular thin film technologies previously developed in our laboratory [38- 
42]. Cantilever bending radius, tracked optically, was proportional to tissue contractile force. Stiffness and contraction/tone assays were both performed using acute (24 hr) pergolide exposures of $1 \mu \mathrm{M}$ concentration.

\subsection{Aortic Valve Interstitial Cell Source and Isolation}

Primary AVICs were isolated from freshly harvested porcine hearts obtained in compliance with FDA guidelines (Blood Farms Inc., Groton, MA). Aortic valve leaflets were extracted from sacrificed hearts and kept in chilled, sterile phosphate buffered saline solution during transport. To harvest AVICs, intact leaflets were dissected from the whole heart and subjected to sequential collagenase digestions at 10 U/l in Hanks' Balanced Salt Solution (HBSS, 55021C, Sigma Aldrich, St. Louis, MO) for 5 min to remove endothelial cells, followed by a 2.5 $\mathrm{hr}$ collagenase digestion at $37^{\circ} \mathrm{C}$ to dislodge the AVICs from the tissue matrix. AVICs were filtered from the remaining leaflet matrix, centrifuged at 10,000 RPM for $10 \mathrm{~min}$, and cultured in M199 media supplemented with 10\% fetal bovine serum (FBS; Invitrogen, Carlsbad, CA), 20 $\mathrm{mM}$ glucose, $10 \mathrm{U} / \mathrm{ml}$ penicillin/streptomycin, $1.5 \mu \mathrm{M}$ vitamin B12, $10 \mathrm{mM}$ HEPES, and 0.1 $\mathrm{mM}$ non-essential amino acids. AVICs were cultured in T75 culture flasks coated with $1 \%$ porcine gelatin (G2500, Sigma-Aldrich, St. Louis, MO). At passage 2, AVICs were suspended in a 90/10 solution of FBS/DMSO and cryopreserved in liquid nitrogen.

\subsection{Engineered Valve Tissues}

To recapitulate aortic valve leaflet structure in vitro (Fig. 1Ai), we first fabricated fibrous polymer/bioprotein ("biohybrid") tissue engineering scaffolds using Rotary Jet Spinning [43-46]. Scaffold composition and fiber diameter specifications were formulated and sized based upon tissue engineered valve constructs previously reported in the literature [47-49]. Scaffold material precursors consisted of poly-4-hydroxybutyrate (55\%, Tepha Inc., Lexington, MA), porcine 
gelatin (40\%, G2500, Sigma-Aldrich), and polyglycolide (5\%, 457620, Sigma-Aldrich, St. Louis, MO) homogenously mixed in hexafluoroisopropanol (003409, Oakwood Chemical, West Columbia, SC) at 4\% w/v prior to fabrication. Fibers were extruded at 30,000 RPM and collected onto rotating cylindrical mandrels at 3,000 RPM to produce anisotropically aligned scaffold sheets approximately $200 \mu \mathrm{m}$ thick; sheets were composed of fibers having an average diameter of approximately $1 \mu \mathrm{m}$ (Fig. 1Bi). The composition and axial alignment of these scaffolds recapitulated the biaxial stiffness [50] and structural organization of the native valve leaflet ECM.

AVICs were seeded at a density of $200 \mathrm{k}$ cells $/ \mathrm{cm}^{2}$ onto $8 \times 8 \mathrm{~mm}$ scaffold pieces cut with the squared faces parallel and perpendicular to the primary axis of fiber alignment (for all conditions and directions $\mathrm{n}=5$ tissues). AVICs were then cultured for $48 \mathrm{hr}$ in growth medium followed by a serum-free drug incubation period of $24 \mathrm{hr}$ as detailed in Section 2.4 (Fig. 1Bii). After the drug incubation period, seeded scaffold pieces were individually loaded onto a biomaterials mechanical tester (BioTester, CellScale Inc., Waterloo, ON) and subjected to equibiaxial tensile loading with applied force ramped linearly between 0 and $2500 \mathrm{mN}$ at a rate of $1 \mathrm{~Hz}$ (Fig. 1Biii). Force-displacement measurements and corresponding images were recorded at $15 \mathrm{~Hz}$. Tensile elastic modulus, E, was calculated using the slope of the tangent in the linear portion of the stress vs. strain curve and the ultimate tensile strength (UTS) was taken to be the largest stress value obtained prior to failure.

To visualize tissue formation, AVICs cultured on the biohybrid scaffolds were imaged using scanning electron microscopy (SEM). After the $24 \mathrm{hr}$ drug incubation, tissues were fixed in 2.5\% glutaraldehyde (G7776, Sigma-Aldrich) in 0.1 M HEPES (15630-080, Invitrogen, Carlsbad, CA) for $1 \mathrm{hr}$. Samples were then rinsed 3x in 0.1 M HEPES and 3x in D.I. water for 5 
min each. Immediately following rinsing, samples were serially dehydrated in $30 \%, 50 \%, 70 \%$, 90\%, and 3x 100\% ethanol (89125, VWR, Randor, PA) washes for 5 min each. Samples were dried for SEM imaging using a critical point drier (931 Series SAMDRI, Tousimis, Rockville, MD) and sputter coated in $5 \mathrm{~nm}$ of 80/20 platinum/palladium (EMS 300TD, Quorum Technologies, Lewes, East Sussex) to prevent charge accumulation and sample degradation during imaging. A field emitting electron microscope at $15 \mathrm{kV}$ (FESEM Ultra Plus, Carl Zeiss, Oberkochen, Germany) with a high efficiency secondary electron detector was used to image samples.

\subsection{AVIC Tissue Tone Stress Assay}

Based on muscular thin film (MTF) technology developed in our laboratory [38-42], we engineered tissues of anisotropically patterned AVICs to produce contractile thin films. AVIC tissue alignment was engineered to recapitulate the native leaflet's circumferential alignment in the fibrosa layer of the leaflet. PDMS thin films were fabricated using a spin coating and laser cutting processes previously reported by our laboratory [40]. Briefly, $22 \times 22 \mathrm{~mm}$ glass coverslips (Product\# 260300, Ted Pella Inc. Redding CA) served as the substrate of the thin film chips and were covered in a low adhesion tape for masking (Product\# 2080, 3M, St. Paul, MN). Two rectangular island shapes with rounded edges of dimensions $18 \times 5 \mathrm{~mm}$ were cut from the tape with a $\mathrm{CO}_{2}$ laser (Epilogue, Golden, $\mathrm{CO}$ ) and removed with forceps (Fig. 2Ai); all laser cutting protocols generated in CorelDRAW (Corel Inc., Ottawa, ON). The thermosensitive polymer poly(N-isopropylacrylamide), PIPAAm, (Polysciences Inc., Warrington, PA) at 10\% w/v in butanol was spin coated at 6000 RPM for 1 min onto masked coverslips with the rectangular islands removed (G3P8 Specialty Spin Coater, SCS Inc., Indianapolis, IN) to create PIPAAm islands (Fig. 2Aii). Following PIPAAm spin coating, the masking was removed and 
polydimethylsiloxane, PDMS, (Slygard 184 elastomer, Dow Corning, Midland, MI) mixed at a 10:1 base to curing agent ratio was spin coated over the PIPAAm islands at 5000 RPM for 5 min to achieve a uniform $18 \mu \mathrm{m}$ coating, (Fig. 2Aiii) as measured using a contact profilometer (Dektak 6M, Veeco Instruments Inc., Plainview, NY). Once cured, 1x3 mm cantilevers were cut into cured thin film chips over the PIPAAm islands with a $\mathrm{CO}_{2}$ laser (Fig. 2Aiv).

To mimic the circumferential fibrosa alignment of the native valve, AVIC tissues were aligned in the direction of PDMS cantilevers using microcontact printing techniques. $20 \mu \mathrm{m}$ lines of human fibronectin (BD Biosciences, Sparks, MD) separated by a gap of $20 \mu \mathrm{m}$ were microcontact printed using PDMS stamps incubated for $1 \mathrm{hr}$ with $50 \mu \mathrm{g} / \mathrm{ml}$ of fibronectin in D.I. water. After stamp incubation, chips were exposed to UV-ozone (Model\# 342, Jetlight Company Inc., Phoenix, AZ) for 8 min then stamped with air-dried fibronectin coated stamps; stamps were brought in contact with chips for less than 1 min. Following fibronectin stamping, chips were immersed in a $5 \mu \mathrm{g} / \mathrm{ml}$ solution of fibronectin in D.I. water for $15 \mathrm{~min}$ to allow for a background coating of fibronectin between the stamped lines (Fig. 2Av).

AVICs were seeded onto stamped chips at a density of $100 \mathrm{k}$ cells $/ \mathrm{cm}^{2}(1 \mathrm{M}$ cells $/$ thin film chip in a standard 6 well plate) and statically cultured for $48 \mathrm{hr}$ in standard growth media detailed above in Section 2.1 (Fig. 2Avi, $n=19$ tissues for $1 \mu \mathrm{M}$ pergolide and $\mathrm{n}=18$ tissues for no-drug). After $48 \mathrm{hr}$, AVIC tissues were rinsed in warmed phosphate buffered saline (PBS) at $37^{\circ} \mathrm{C}$ to remove potential residual serotonin, dopamine, or other potential confounding factors present in the media serum that may influence drug testing and cultured in serum-free media for $24 \mathrm{hr}$. During the $24 \mathrm{hr}$ serum-free incubation, thin film chips were subjected to drug treatment of $1 \mu \mathrm{M}$ pergolide or no-drug (pergolide: P8828, Sigma-Aldrich, St. Louis, MO). A $1 \mu \mathrm{M}$ dose of pergolide is a saturating concentration for stimulation of the $5 \mathrm{HT}-2 \mathrm{~B}$ receptors of heart valve 
interstitial cells $[51,52]$ and is an approximation of the blood plasma concentration in the body of an adult patient taking 1-5 $\mathrm{mg}$ of pergolide daily, a clinically relevant dosing range [6,33].

After the $24 \mathrm{hr}$ serum-free drug treatment, thin film chips were gently rinsed with and placed into $5 \mathrm{ml}$ of Tyrode's solution at $37^{\circ} \mathrm{C}$; all experiments were performed in $37^{\circ} \mathrm{C}$ warmed Tyrode's solution (1.192 g/l HEPES, 0.040 g/l NaH $\mathrm{NO}_{4}, 0.901 \mathrm{~g} / \mathrm{l}$ glucose, $0.265 \mathrm{~g} / \mathrm{l} \mathrm{CaCl}$, $0.203 \mathrm{~g} / \mathrm{l} \mathrm{MgCl}, 0.403 \mathrm{~g} / \mathrm{l} \mathrm{KCl}, 7.889 \mathrm{~g} / \mathrm{l} \mathrm{NaCl}, \mathrm{pH}$ adjusted to 7.4 using $1 \mathrm{~N} \mathrm{NaOH}$, all chemicals from Sigma-Aldrich, St. Louis, MO). To lift the PDMS cantilevers, chips in Tyrode's solution were allowed to briefly cool below $32^{\circ} \mathrm{C}$, allowing PIPAAm to phase change into a hydrophilic state, thus releasing the cantilevers which were carefully peeled free from the PIPAAm islands with forceps (Fig. 2Bi). Once the cantilevers were released, the tissue tone assay was performed on a stereomicroscope (Fig. 2Bii, Model SteREO Discovery.v12, Zeiss).

During the assay, images were taken directly above the thin film chip (Fig. 2Biii) every $30 \mathrm{sec}$. For the first $5 \mathrm{~min}$ of the assay, thin films were allowed to equilibrate and establish a basal tone tissue stress. Subsequently, AVIC tissue active contraction was induced via the known vasoconstrictor endothelin-1 (Et-1, E7764, Sigma-Aldrich, St. Louis, MO), at a $100 \mathrm{nM}$ saturating concentration for $15 \mathrm{~min}$. Next, cantilevers were completely relaxed with the addition of the rho-kinase inhibitor HA-1077 dihydrochloride (H139, Sigma-Aldrich) for $10 \mathrm{~min}$ at a 100 $\mu \mathrm{M}$ saturating concentration. Bending of the cantilevers as a result of basal tone and active

contraction was detected using custom Image $\mathrm{J}$ (NIH, Bethesda, MD) software; radius of curvature and stress in the cantilever was then calculated using previously reported custom MATLAB code (Mathworks, Natick, MA) and models [38-42].

\subsection{Immunostaining and Tissue Alignment Analysis}

PDMS-coated coverslips were microcontact printed with $20 \times 20 \mu \mathrm{m}$ fibronectin lines, 
seeded with AVICs, and cultured as described above (Section 2.3). Following drug incubation, coverslips were fixed in 4\% paraformaldehyde (PFA, 15710, Electron Microscopy Sciences, Hatfield, PA) and 0.5\% TritonX 100 (T8787, Sigma- Aldrich, St. Louis, MO) in PBS for 15 min. Coverslips were then rinsed 3x in PBS for 10 min each and incubated in 5\% w/v bovine serum albumin (BSA, 001-000-162, Jackson ImmunoResearch, West Grove, PA) in PBS for nonspecific blocking. After the BSA block, coverslips were incubated in $200 \mu \mathrm{l}$ of $0.5 \% \mathrm{w} / \mathrm{v}$ BSA solution in PBS containing $2 \mu$ of monoclonal anti-alpha smooth muscle actin (mouse) and polyclonal anti-cofilin (rabbit) antibodies (ab7817 and ab11062 respectively; Abcam, Cambridge, Ma) for $2 \mathrm{hr}$. Following primary incubation, coverslips were rinsed 3x in PBS for 10 min each then incubated in $200 \mu 1$ of $0.5 \% \mathrm{w} / \mathrm{v}$ BSA solution in PBS containing $5 \mu 1$ of DAPI, 2 $\mu 1$ Alexa Fluor 633-conjugated phalloidin (A22284, Invitrogen, Carlsbad, CA), $2 \mu 1$ goat antimouse Alexa Fluor-488 (A-11001, Invitrogen, Carlsbad, CA), and $2 \mu$ donkey anti-rabbit Alexa Fluor-546 (A-10040, Invitrogen, Carlsbad, CA). Coverslips were mounted on standard microscope slides and imaged using a Zeiss LSM 7 LIVE confocal microscope. All images were taken at 20x magnification producing a field of view of $160 \times 160 \mu \mathrm{m}$; the number of nuclei within each field of view were used to determine tissue cell density. Fluorescent images of the actin cytoskeleton were used to calculate the Orientation Order Parameter (OOP), a relative measure of axial alignment in tissues [53, 54]. The OOP of a tissue is calculated based upon fingerprint identification algorithms which give a score of tissue anisotropy; an OOP score of 1 indicates perfect axial alignment while an OOP score of 0 indicates no preferential axis of alignment ( $\mathrm{n}=8$ tissues, $5 \mathrm{ROI} /$ tissue for tissue cell density and OOP measurements; all reported as mean \pm standard error of the mean). 


\subsection{Western Blot Protein Expression Analysis}

To quantify changes in protein expression indicative of AVIC activation state, tissues microcontact printed and cultured as described above (Section 2.3), were lysed at $4^{\circ} \mathrm{C}$ in RIPA lysis buffer (SLBG8489, Sigma, St. Louis, MO) plus Complete Mini (11836153001, Roche Diagnostic, Mannheim, Germany) and Halt-Protease and Phosphotase Inhibitor (1861281, ThermoFisher Scientific, USA). Protein expression levels were quantified using a capillarybased Wes ${ }^{\text {TM }}$ Simple Western Analysis (ProteinSimple, San Jose, CA). All procedures were performed according to the manufacturer's protocol (cell lysates were pooled from $n=5$ tissues for each condition). Briefly, $1 \mu \mathrm{g}$ cell lysates were loaded into each capillary and proteins were separated by size in the stacking and separation matrix. Target proteins, including $\alpha-S M A$, cofilin, and $\alpha$-tubulin (loading control), were identified using primary antibodies (ab7817, ab11062, and ab7291, respectively, Abcam, Cambridge, MA). The proteins were immunoprobed using manufacturer provided secondary antibody and chemiluminescent substrate. The resulting chemiluminescent signal was detected and quantified using Compass Software (Protein Simple, San Jose, CA).

\subsection{Statistical Analysis}

Changes in tissue stiffness, contractility, and alignment due to pergolide exposure were statistically evaluated using SigmaPlot software (v12.0, Systat Software Inc., San Jose, CA). Tissue moduli and ultimate tensile strength in the parallel and perpendicular directions of scaffold fiber/tissue alignment for no-drug vs. $1 \mu \mathrm{M}$ pergolide (Section 2.2) were compared with t-tests, passing both the Shapiro-Wilk normality and equal variance tests. Active tissue tone stress generation for no-drug vs. $1 \mu \mathrm{M}$ pergolide (Section 2.3) failed the Shapiro-Wilk normality test $(\mathrm{p}<0.05)$ and thus were evaluated using the Mann-Whitney Rank Sum Test. Basal tissue tone 
stress generation for no-drug vs. $1 \mu \mathrm{M}$ pergolide (Section 2.3) passed Shapiro-Wilk normality and equal variance and were thus compared with t-tests. Changes in tissue alignment OOP for all no-drug vs. $1 \mu \mathrm{M}$ pergolide (Section 2.4) were evaluated with t-tests, passing both the ShapiroWilk normality and equal variance tests. For all statistical analyses, p-values less than 0.05 were considered statistically significant.

\section{Results}

\subsection{Pergolide-induced Tissue Stiffening}

The clinical manifestation of pergolide-induced VHD is valvular dysfunction (e.g. regurgitation and/or stenosis) resulting from stiffened fibrotic leaflets [26]. We therefore asked if pergolide-induced AVIC tissue stiffening could be measured in vitro using biaxial tensile testing of engineered AVIC tissues. AVIC tissues were engineered on valve-like scaffolds to mimic the fibrosa layer of the leaflet and exposed to $1 \mu \mathrm{M}$ pergolide for $24 \mathrm{hr}$ following a $48 \mathrm{hr}$ period of tissue formation (Fig. 3A). After exposure, tissues were equibiaxially strained and forcedisplacement data were used to measure pergolide-induced changes in tissue tensile modulus, E, and UTS (Fig. 3B). E values measured in the primary axis of fiber/tissue alignment (parallel) increased by $27 \%$ following $24 \mathrm{hr}$ exposure to $1 \mu \mathrm{M}$ pergolide (t-test $\mathrm{p}<.05$, Fig. 3Ci). E values in the perpendicular axis of fiber/tissue alignment increased by $23 \%$ following $24 \mathrm{hr}$ exposure to $1 \mu \mathrm{M}$ pergolide (t-test $\mathrm{p}=0.49$, Fig. 3Cii). No differences in UTS were observed. These data suggest a directionally dependent increase in tensile modulus (stiffness) along the axis of tissue alignment resulting from acute $(24 \mathrm{hr})$ pergolide exposure.

\subsection{Pergolide-induced Reduction of Tissue Tone Generation and Structural Anisotropy}

The increased tensile modulus that we observed in AVIC tissues exposed to an acute pergolide dose suggests that pergolide induced a phenotypic change towards synthetic AVIC 
tissues. We therefore asked if a change from the fibroblast-to-myofibroblast phenotypic activation state in AVIC tissues was induced in response to acute pergolide exposure. Immunostaining and protein quantification of AVIC activation markers revealed expression of both $\alpha$-SMA and cofilin in both no-drug and pergolide treated tissues. $\alpha$-SMA staining in nodrug AVIC tissues appeared more continuous and uniform throughout the tissue when compared to pergolide treated tissues. Cofilin staining in no-drug AVIC tissues appeared more diffuse compared to pergolide treated tissues (Fig. 4Ai), where cofilin revealed a high degree of colocalization with cytoskeletal f-actin (Fig. 4Aii). Western blot protein expression showed a 30\% decrease in $\alpha$-SMA expression in pergolide treated AVIC tissues, whereas cofilin expression was unchanged by drug exposure (Fig. 4B). Given that AVIC activation has been shown to decrease with increased cell density [55], our data taken from confluent tissues suggest that pergolide exposure increases this contact driven activation-to-quiescence mechanism acutely in AVICs.

We then asked if this phenotypic shift would affect the AVIC tissue's ability to modulate tissue tone. To measure tissue tone in vitro, anisotropic AVIC tissues were engineered using microcontact printing on thin film cantilever substrates; after $48 \mathrm{hr}$ of growth, tissues were exposed to $1 \mu \mathrm{M}$ pergolide for $24 \mathrm{hr}$. Following exposure, thin film tissues were released at one end permitting optical measurement of active and basal tissue tone stress generation (Fig. 5A). Released cantilevers were given 5 min to acclimate and reach their basal tone (Fig. 5Bi) followed by $15 \mathrm{~min}$ of Et-1 induced active contraction (Fig. 5Bii) and $10 \mathrm{~min}$ of HA-1077 induced relaxation (Fig. 5Biii). Although AVIC tissues in both no-drug and $1 \mu \mathrm{M}$ pergolide treated groups were equally dense (Fig. 5Ci), pergolide exposed tissues had a significantly reduced active and basal tone stress generation (t-test $\mathrm{p}<.05$, Fig. 5Cii active and 5Ciii basal). Because of the unidirectional nature of our contractility assay, we asked if these observed losses in active 
and basal tissue tone were influenced by changes in tissue anisotropy, i.e. deviation in alignment relative to the contractile direction of the thin films. As measured by their f-actin OOP, $1 \mu \mathrm{M}$ pergolide treated tissues had a reduction in tissue alignment of $7 \%$ relative to no-drug controls (ttest $\mathrm{p}<.05$, Fig. 5D). Taken together, these data suggest that acute pergolide exposure reduces AVIC tissue stress generation capacity, which is due, at least in part, to a loss of axial tissue alignment.

\section{Discussion}

Using engineered AVIC tissues exposed to an acute pergolide dose, we observed a significant increase in tissue tensile modulus along the primary axis of fiber/tissue alignment. This increase in tensile modulus of our thin AVIC tissue model is suggestive of the drastic increases in stiffness observed clinically in cases of valve fibrosis. Fibrotic valve lesions observed in patients pathologically affected by pergolide are accompanied by leaflet tissue stiffening that ultimately leads to valvular insufficiency $[9,56]$. The current standard of care for monitoring drug-induced functional heart valve irregularity is echocardiography, usually performed at roughly 6 month intervals [57]. Our results indicate that pathological changes in biaxial valve tissue stiffness can potentially be observed and quantitatively measured on the order of days rather than months. Future studies may determine whether early onset stiffness changes can be observed clinically using high resolution echocardiography [58] or MRI-based [59] non-invasive imaging techniques.

Our results support the hypothesis that tissue-level pergolide-induced pathologies may, at least in part, be due to a shift in AVIC phenotype towards an overly synthetic activation state [60]. This was further evident in the effect of pergolide on our contractile thin film assay; that is, stiffer and more synthetic AVIC tissues were not able to generate as much tissue tone. When 
treated with the same pergolide dose $(1 \mu \mathrm{M})$, the thin film tissue stress assay showed a nearly $50 \%$ reduction in both active and basal tone stress generation. The significantly reduced contractility following drug exposure is further suggestive of the potential off-target effect of pergolide on the cardiac valves. In particular, as AVICs lose their stress generation capacity, they may lose their ability to maintain tissue tone within the leaflet, thus disrupting their homeostatic mechanical environment. A loss in this mechanical feedback mechanism may result in further and more permanent AVIC pathological activation within the tissue [61].

$\alpha$-SMA and cofilin staining and expression quantification were consistent with the observed AVIC tissue tone stress generation data. The expression of $\alpha$-SMA, a protein critical to the contractile mechanism of AVICs, was decreased in pergolide treated tissues, which is consistent with the observed decrease in active and basal stress generation relative to no-drug treated tissues. Additionally, increased cofilin-actin colocalization in pergolide treated tissues suggests increased cell motility within the tissue [62] which is an indicator of early myofibroblast response to injury [63]. Colocalization of cofilin with cytoskeletal f-actin is necessary for eventual stress fiber assembly [64] and has been proposed to be required for cardiac myofibroblast differentiation after force-induced injury of the myocardium [65]. As a result, the initial decrease in $\alpha$-SMA expression and increase in actin-colocalization of cofilin in pergolide treated AVIC tissues observed here may reflect early "proto-myofibroblast" stages of permanent myofibroblast differentiation in our engineered tissues.

Additionally, the loss of functional tissue tone generation in our contractility assay is indicative of a loss in the AVIC population's stress generation capacity in a single direction. Because our AVIC tissues were engineered to be initially aligned in the direction of the thin film cantilevers, a loss in tissue alignment will reduce measured contractile stresses. We observed a 
$7 \%$ loss of AVIC alignment in response to $1 \mu \mathrm{M}$ pergolide exposure, as quantified using the orientation order parameter. The pergolide-induced reduction in measured AVIC tissue contractility therefore likely resulted from increased tissue stiffness, early reduction in $\alpha$-SMA expression, and loss of tissue alignment. This is in agreement with previously reported mathematical models of varied tissue orientation and thin film mechanics [66] as well as druginduced changes in tissue alignment that influenced smooth muscle cell stress generation [67].

The standard of any in vitro drug screening platform or Organ-on-a-Chip technology [68, 69] is its relevance to the clinic. In the case of this study, the retrospective and pathological comparisons of pergolide-induced VHD observed clinically and those observed using our platforms must be critically compared. We aimed to expand upon the current mechanistic study of drug-induced VIC pathology by developing tissue-scale mechanical models. The AVIC tissue assays we built showed pronounced functional changes in AVIC tissue tone generation, loss of tissue alignment, and a significant increase in tissue tensile modulus following acute drug exposure. In particular, the tissue modulus and alignment results draw direct comparison to the gross structural and mechanical changes of pergolide-induced VHD observed clinically and in explanted tissue [70]. At the cellular-scale, markers of off-target cardiac valve pathologies induced by pergolide and other ergoline-derived medications may include morphological changes in tissue alignment or other biological markers such increased chronic $\alpha$-SMA expression and excessive mitogenic activity [71]. However, we additionally propose that an increase in stiffness and loss of tone generation capacity at the tissue-scale may serve as effective functional markers of early drug-induced VHD for current and future neurological medication evaluation and development. Many non-specific dopamine agonists including the drugs 3,4methylenedioxymethamphetamine (MDMA) [51], fenfluramine-phentermine [7, 72], and 
cabergoline and pergolide $[9,25,73]$ have been shown to cause valvular dysfunction. Similar new dietary, Parkinson's disease, and other psychiatric drug development designed to act on serotonin and/or dopamine may benefit from these platforms as techniques to determine early warning signs of VHD. The in vitro assays presented here are well-suited to study AVIC activation and tissue-level consequences of AVIC-myofibroblast dysregulation and differentiation. We expect further use of these assays to identify off-target drug effects that alter the phenotypic balance of AVICs, disrupt their ability to maintain mechanical homeostasis, and lead to VHD [10, 15, 16, 74].

\section{Conclusion}

We designed and built two complimentary in vitro assays to measure AVIC tissue stiffness and contractile capacity, and validated their use for preclinical drug screening using a known valvulopathogen, pergolide. Our contractile thin film tissue tone assay was sufficiently sensitive to reveal pergolide-induced reduction of AVIC tissue contractile tone concomitant with increased tissue stiffness and loss of tissue anisotropy measured independently. These results highlight the role of AVICs in the maintenance of tissue tone, are suggestive of the acute offtarget effects of pergolide and, potentially, other 5HT-2B receptor modulators. Because our assays are based on measurements of tissue-level pathologies, they will serve as effective preclinical drug screening assays that bridge cell-based assays with clinical evaluation methods.

Study Limitations: This study was limited to acute, $24 \mathrm{hr}$, drug exposure; therefore, these data are indicative of the very early onset of off-target drug effects on the cardiac valves, of which we have little direct clinical comparison. Therefore, these results are suggestive of the chronic pathologies observed clinically and further time points are required to investigate the full etiology of ergoline-derived drug-induced VHD. Additionally, all samples were cultured under 
static conditions thus not mimicking the pulsatile flows and pressures that the native valve is exposed to. The native valve is largely composed of VICs covered by a monolayer of valvular endothelial cells (VECs) that regulate VIC phenotype [75]. Although we chose to study AVICs in isolation, future studies will include VIC/VEC co-cultures.

\section{Acknowledgements}

We thank the Harvard Center for Nanoscale Systems (CNS) for the use of cleanroom facilities in the fabrication of our organ-chips and imaging of scaffold, the Wyss Institute of Biologically Inspired Engineering, Kartik Balachandran for sharing his expertise in cardiac valve cell isolation/culture, Peyton Nesmith with for his thin film assay expertise and clinical input, and Alexander Cho for assisting with thin film fabrication. This work was funded by the National Institutes of Health (4UH3TR000522-03) and National Science Foundation (MRSEC NSF DMR-1420570). 


\section{Figure 1}

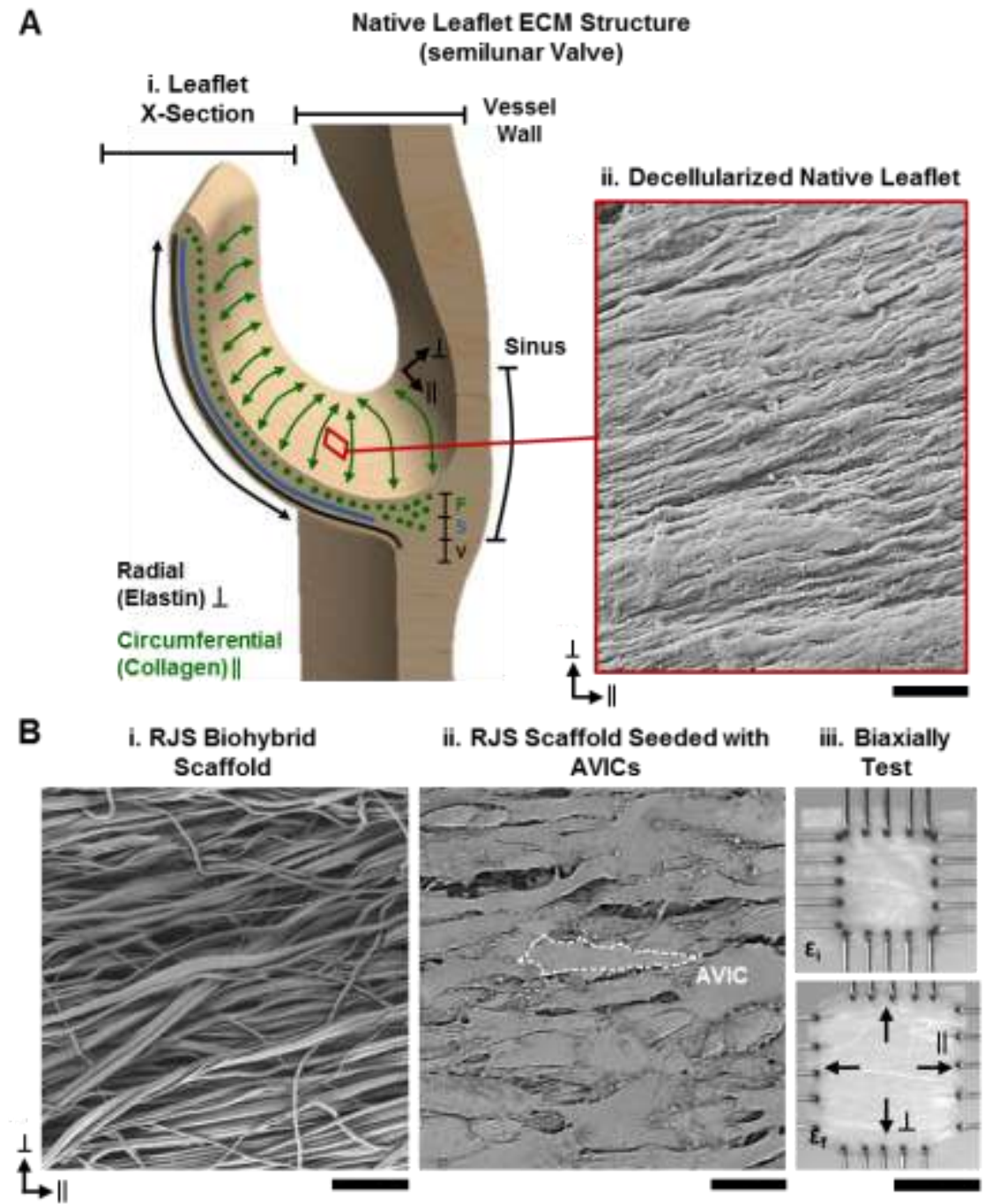

Fig. 1. Biohybrid scaffolds fabricated using the Rotary Jet Spinning (RJS) system were designed to mimic the circumferential alignment of the valve fibrosa extracellular matrix (ECM, A). The circumferentially oriented fibrosa "F" provides the mechanical strength of the leaflet while the spongiosa " $\mathrm{S}$ " is thought to provide cushioning during leaflet closure during diastole and the elastin-rich ventricularis "V" provides recoil after leaflet opening during systole ( $\mathrm{A}$, leaflet cross-section). The inset to Aii shows a scanning electron microscopy (SEM) image of decellularized porcine fibrosa (Aii, SEM, scale bar $5 \mu \mathrm{m}$ ), which was recapitulated using RJS produced nanofibers. Circumferentially oriented nanofibers (Bi, SEM, scale bar 20 $\mu \mathrm{m}$ ) were seeded with AVICs and allowed 48 hrs of growth to form a confluent tissue (Bii, SEM, scale bar 20 $\mu \mathrm{m})$ followed by $24 \mathrm{hrs}$ of pergolide or no-drug exposure. After exposure, AVIC tissues were equibiaxially strained (Biii, optical images, scale bar $5 \mathrm{~mm}$ ) to determine drug induced changes in tissue level stiffness and strength $\left(\varepsilon_{\mathrm{i}}\right.$ : initial strain at $.05 \%$, $\varepsilon_{\mathrm{f}}$ : final strain at $\left.40 \%\right)$. 
Figure 2

A

AVIC Tissue Contractility Assay Fabrication

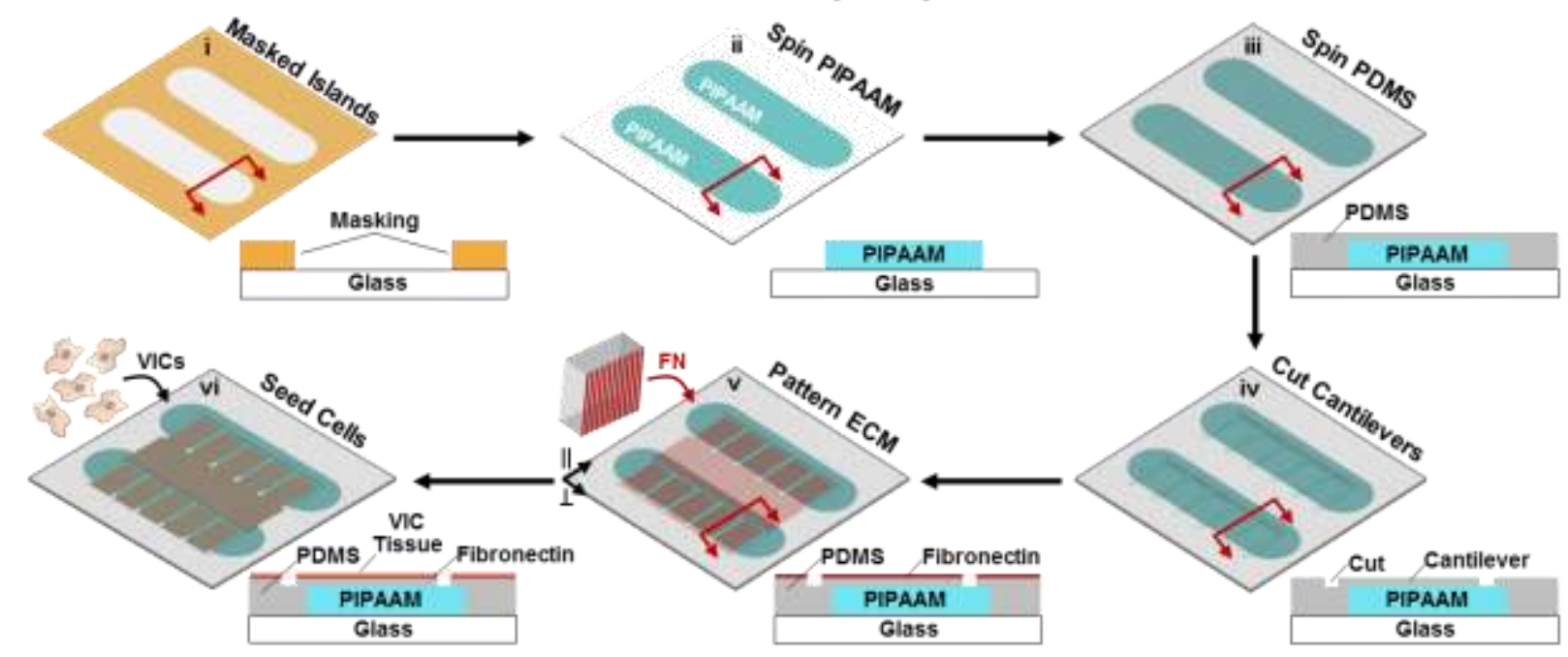

B

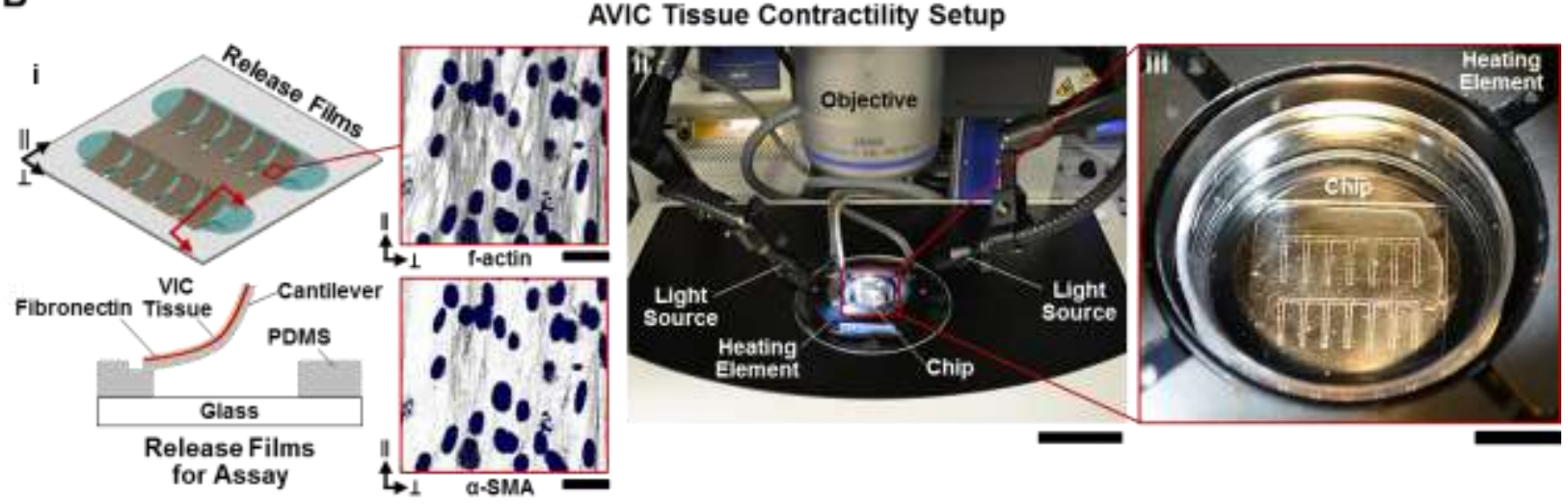

Fig. 2. The contractile thin film assay was designed to recapitulate the circumferential alignment of the fibrosa layer of the native leaflet ECM in 2D. The fibrosa is primarily composed of fibrous collagen bundles and cellbinding proteins such as fibronectin. To fabricate releasable thin films, a sacrificial layer of PIPAAM was spin coated into islands on a glass coverslip (Ai: masked islands, Aii: spin-coated PIPAAM within islands), followed spin coating a thin layer of PDMS (Aiii) that was cured and laser-cut into cantilevers (Aiv). AVIC tissues aligned in the direction of the cantilevers were created by microcontact printing 20x20 $\mu \mathrm{m}$ lines of fibronectin (Av) and seeding AVICs onto the chips (Avi). AVIC tissues were allowed a $48 \mathrm{hr}$ growth period followed by a $24 \mathrm{hr}$ pergolide or no-drug exposure. Following drug exposure, cantilevers were released via temperature driven PIPAAM dissolution (B). Bi shows a schematic of thin film release (left) and the insets (right) show confluent, aligned VICs (immuno-micrographs, scale bars $25 \mu \mathrm{m}$, blue: DAPI, black top: f-actin, black bottom: $\alpha$-SMA). Thin film bending radius was tracked optically using a stereomicroscope and CCD (Bii, optical images, scale bar is $10 \mathrm{~cm}$; Biii inset: thin film chip, scale bar is $5 \mathrm{~cm}$ ). 


\section{Figure 3}
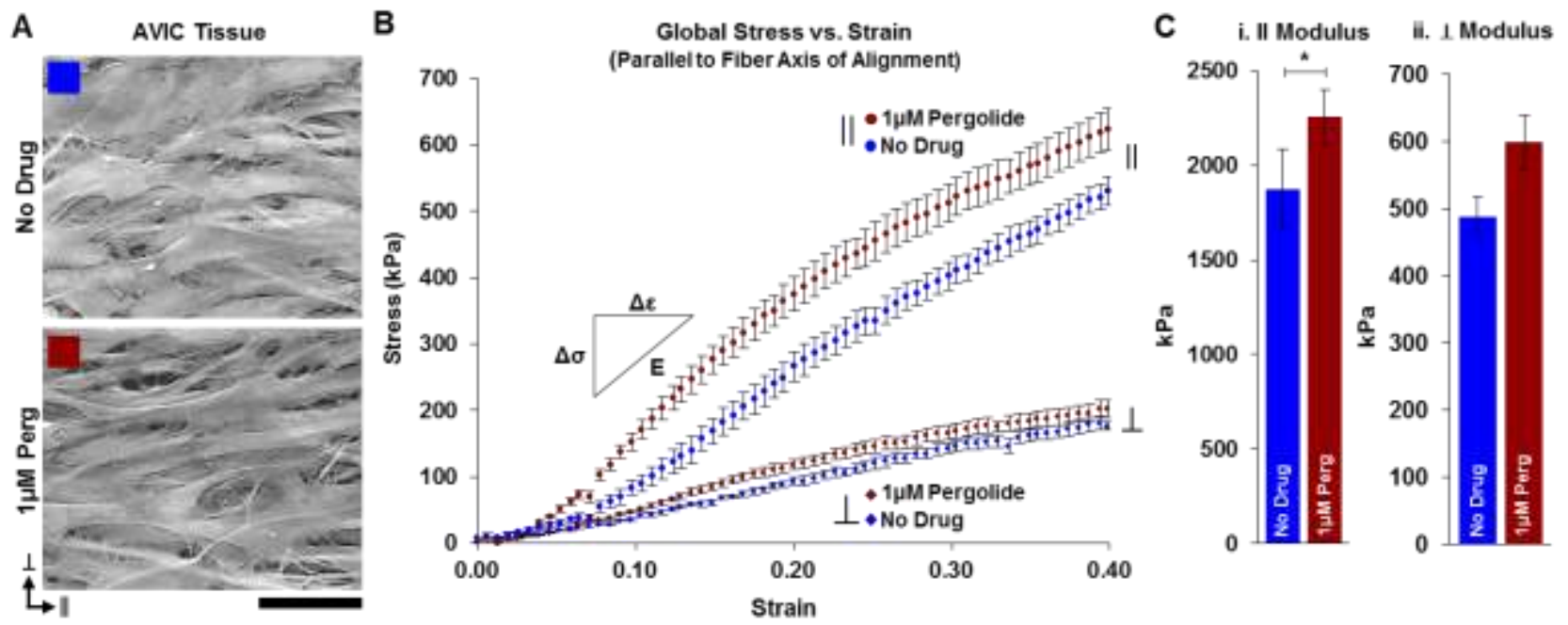

Fig. 3. Representative scanning electron microscopy images of AVIC tissues cultured on biohybrid scaffolds (A, scale bar is $25 \mu \mathrm{m}$ ). Engineered AVIC tissue tensile moduli (E) values were obtained from the global tissue stress $(\sigma)$ vs. strain $(\varepsilon)$ curves of samples strained both parallel and perpendicular to fiber/tissue alignment (B, error is standard error of the mean). Tissues exposed to $1 \mu \mathrm{M}$ pergolide had an average parallel modulus of $2,254.24 \pm 146.67 \mathrm{kPa}(\mathrm{Ci}$, red) and average perpendicular modulus of $598.56 \pm 39.49 \mathrm{kPa}$ (Cii, red). No-drug control tissues had an average parallel modulus of 1,773.52 $\pm 83.12 \mathrm{kPa}(\mathrm{Ci}$, blue $)$ and average perpendicular modulus of $486.56 \pm 31.53 \mathrm{kPa}$ (Cii, blue). For all conidtions/directions $\mathrm{n}=5$, reported as mean \pm standard error of the mean, $* \mathrm{p}<0.05$. 


\section{Figure 4}

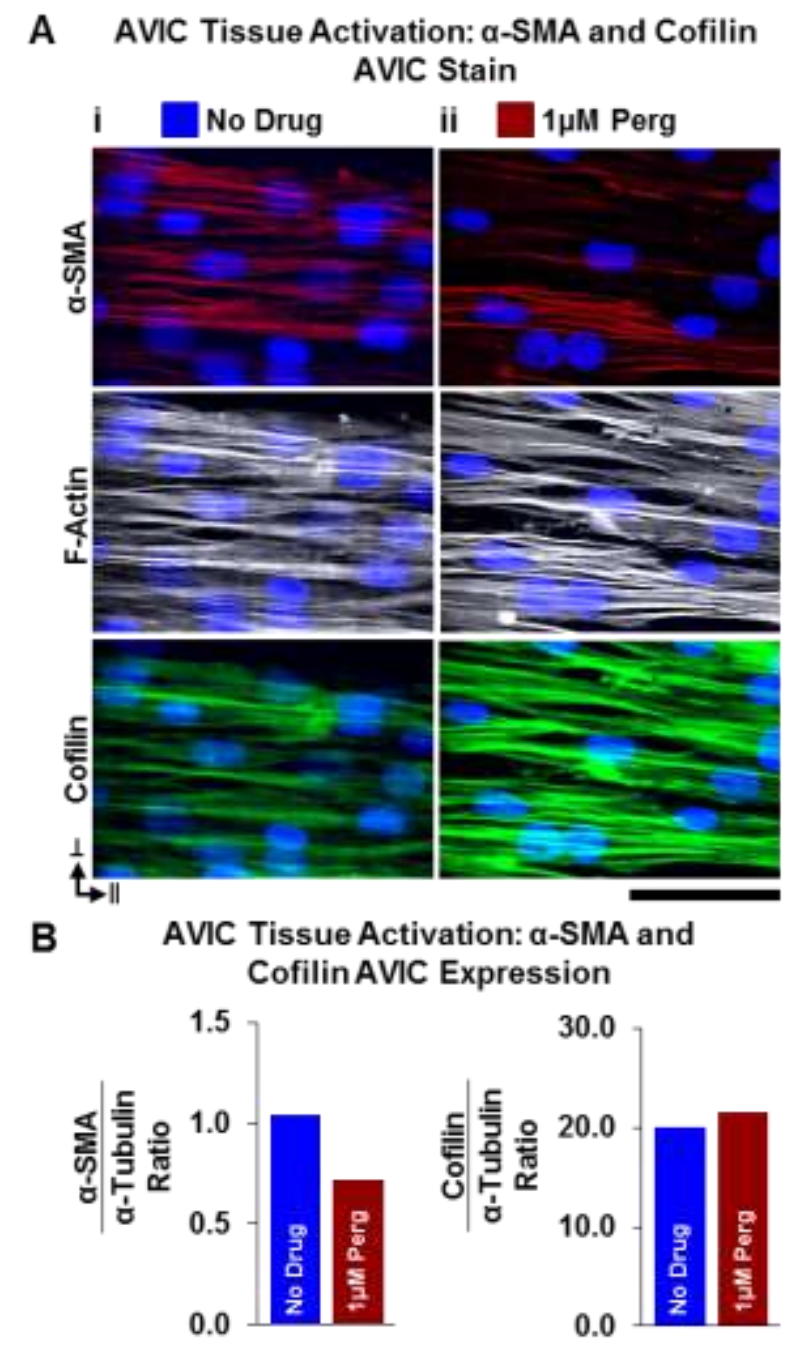

Fig. 4. No-drug and pergolide treated AVIC tissues expressed both alpha smooth muscle actin ( $\alpha$-SMA) and cofilin. No-drug AVIC tissues exhibited more uniform $\alpha$-SMA staining throughout the tissue (Ai, red) while cofilin staining was more diffuse within cells (Ai, green). $1 \mu \mathrm{M}$ pergolide treated tissues exhibited less pronounced $\alpha$-SMA staining throughout tissues (Aii, red) but a higher degree of cofilin-actin colocalization (Aii, white: f-actin and green: cofilin) than no-drug tissues (scale bar $50 \mu \mathrm{m}$ for all images; $\mathrm{n}=3$ tissues per condition). Protein quantification revealed a $30 \%$ relative decrease in $\alpha$-SMA expression due to acute pergolide exposure while no difference was observed in relative cofilin expression due to the drug (B, cell lysates were pooled from $n=5$ tissues for each condition, proteins of interest normalized to $\alpha$-tubulin as the loading control). 
Figure 5
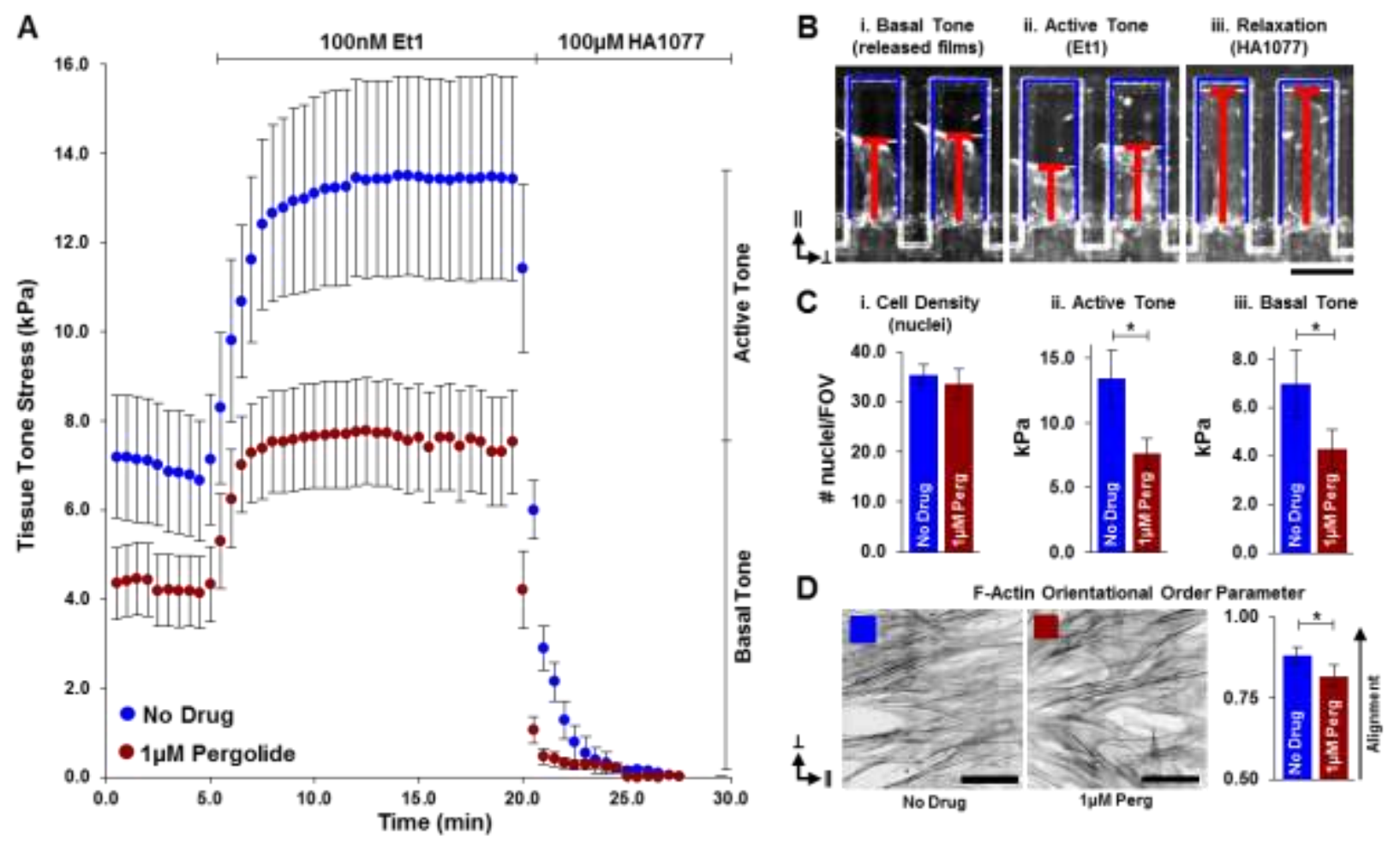

Fig. 5. Following AVIC tissue growth, exposure, and thin film release the tissue tone generation assays were conducted (A). First, a basal tone was established for $5 \mathrm{~min}$ (Bi) followed by the induction of active stress generation via administration of $100 \mathrm{nM}$ endothelin-1 (Et-1, Bii). Following 15 min of active contraction, 100 $\mu$ M HA1077 was administered to induce full relaxation of the cantilevers for 10 min (Biii; scale bars in B 1 $\mathrm{mm}$, all optical images). No differences in tissue cell density were observed among the conditions $(\mathrm{Ci}, \mathrm{n}=4$ tissues with $5 \mathrm{FOV} /$ tissue for no-drug tissues and n=5 tissues with $5 \mathrm{FOV} /$ tissue for pergolide treated tissues). Tissues exposed to $1 \mu \mathrm{M}$ pergolide exhibited reduced tissue tone generation of $7.60 \pm 1.19 \mathrm{kPa}$ active tone (Cii, red) and $4.28 \pm 0.80 \mathrm{kPa}$ basal tone (Ciii, red) compared to no-drug controls which exhibited tissue tone generation of $13.40 \pm 2.23 \mathrm{kPa}$ active tone (Cii, blue) and $6.97 \pm 1.37 \mathrm{kPa}$ basal tone (Ciii, blue). All conditions reported as mean \pm standard error of the mean $(n=19$ tissues for $1 \mu \mathrm{M}$ pergolide and $n=18$ tissues for no-drug, ${ }^{*} \mathrm{p}<0.05$ ). To investigate tissue alignment, OOP of the f-actin cytoskeleton were measured (D, immuno-micrographs, scale bars $50 \mu \mathrm{m}$; blue: DAPI, black: f-actin). Tissues exposed to $1 \mu \mathrm{M}$ pergolide exhibited a decreased OOP of $0.82 \pm 0.02$ ( $\mathrm{D}, \mathrm{n}=9$ tissues, $5 \mathrm{ROI} /$ tissue) while no-drug control tissues exhibited an OOP of $0.88 \pm 0.02(\mathrm{D}, \mathrm{n}=8$ tissues, $5 \mathrm{ROI} /$ tissue; all reported as mean \pm standard error of the mean, $* \mathrm{p}<0.05)$. 


\section{References}

[1] Brichta L, Greengard P, Flajolet M. Advances in the pharmacological treatment of Parkinson's disease: targeting neurotransmitter systems. Trends Neurosci 2013;36:543-54.

[2] Celada P, Bortolozzi A, Artigas F. Serotonin 5-HT1A receptors as targets for agents to treat psychiatric disorders: rationale and current status of research. CNS Drugs 2013;27:703-16.

[3] Berger M, Gray JA, Roth BL. The expanded biology of serotonin. Annu Rev Med 2009;60:355-66.

[4] Beaulieu J-M, Gainetdinov RR. The physiology, signaling, and pharmacology of dopamine receptors. Pharmacol Rev 2011;63:182-217.

[5] Sellbach A, Silburn P. Management of Parkinson's disease. Management 2012;35.

[6] Van Camp G, Flamez A, Cosyns B, Weytjens C, Muyldermans L, Van Zandijcke M, et al. Treatment of Parkinson's disease with pergolide and relation to restrictive valvular heart disease. Lancet 2004;363:1179-83.

[7] Connolly HM, Crary JL, McGoon MD, Hensrud DD, Edwards BS, Edwards WD, et al. Valvular heart disease associated with fenfluramine-phentermine. New Engl J Med 1997;337:581-8.

[8] Roth BL. Drugs and valvular heart disease. New Engl J Med 2007;356:6-9.

[9] Antonini A, Poewe W. Fibrotic heart-valve reactions to dopamine-agonist treatment in Parkinson's disease. Lancet Neuro 2007;6:826-9.

[10] Hutcheson JD, Setola V, Roth BL, Merryman WD. Serotonin receptors and heart valve disease-it was meant 2B. Pharmacol Therapeut 2011;132:146-57.

[11] Elangbam CS, Job LE, Zadrozny LM, Barton JC, Yoon LW, Gates LD, et al. 5-hydroxytryptamine (5HT)-induced valvulopathy: compositional valvular alterations are associated with 5HT2B receptor and 5HT transporter transcript changes in Sprague-Dawley rats. Exp Toxilcol Pathol 2008;60:253-62.

[12] Hutcheson JD, Ryzhova LM, Setola V, Merryman WD. 5-HT 2B Antagonism arrests non-canonical TGF- $\beta 1$-induced valvular myofibroblast differentiation. J Mol Cell Cardiol 2012;53:707-14.

[13] Chen J-H, Yip CYY, Sone ED, Simmons CA. Identification and characterization of aortic valve mesenchymal progenitor cells with robust osteogenic calcification potential. Am J Pathol 2009;174:110919.

[14] Mulholland DL, Gotlieb AI. Cardiac valve interstitial cells: regulator of valve structure and function. Cardiovasc Pathol 1997;6:167-74.

[15] Liu AC, Joag VR, Gotlieb AI. The emerging role of valve interstitial cell phenotypes in regulating heart valve pathobiology. Am J Pathol 2007;171:1407-18.

[16] Aikawa E, Whittaker P, Farber M, Mendelson K, Padera RF, Aikawa M, et al. Human Semilunar Cardiac Valve Remodeling by Activated Cells From Fetus to Adult Implications for Postnatal Adaptation, Pathology, and Tissue Engineering. Circulation 2006;113:1344-52.

[17] Filip D, Radu A, Simionescu M. Interstitial cells of the heart valves possess characteristics similar to smooth muscle cells. Circ Res 1986;59:310-20.

[18] Walker GA, Masters KS, Shah DN, Anseth KS, Leinwand LA. Valvular myofibroblast activation by transforming growth factor- $\beta$ implications for pathological extracellular matrix remodeling in heart valve disease. Circ Res 2004;95:253-60.

[19] Cushing MC, Liao J-T, Anseth KS. Activation of valvular interstitial cells is mediated by transforming growth factor- $\beta 1$ interactions with matrix molecules. Matrix Biol 2005;24:428-37.

[20] Merryman WD, Youn I, Lukoff HD, Krueger PM, Guilak F, Hopkins RA, et al. Correlation between heart valve interstitial cell stiffness and transvalvular pressure: implications for collagen biosynthesis. Am J Physiol-Heart C 2006;290:H224-H31.

[21] Duan B, Hockaday LA, Kapetanovic E, Kang KH, Butcher JT. Stiffness and adhesivity control aortic valve interstitial cell behavior within hyaluronic acid based hydrogels. Acta biomaterialia 2013;9:764050.

[22] Butcher JT, Nerem RM. Valvular endothelial cells regulate the phenotype of interstitial cells in coculture: effects of steady shear stress. Tissue Eng 2006;12:905-15. 
[23] Butcher JT, Simmons CA, Warnock JN. Review-Mechanobiology of the aortic heart valve. J Heart Valve Dis 2008;17.

[24] Wyss K, Yip CY, Mirzaei Z, Jin X, Chen J-H, Simmons CA. The elastic properties of valve interstitial cells undergoing pathological differentiation. J Biomech 2012;45:882-7.

[25] Schade R, Andersohn F, Suissa S, Haverkamp W, Garbe E. Dopamine agonists and the risk of cardiac-valve regurgitation. New Engl J Med 2007;356:29-38.

[26] Zanettini R, Antonini A, Gatto G, Gentile R, Tesei S, Pezzoli G. Valvular heart disease and the use of dopamine agonists for Parkinson's disease. New Engl J Med 2007;356:39-46.

[27] Zeng Q, Song R, Ao L, Weyant MJ, Lee J, Xu D, et al. Notch1 promotes the pro-osteogenic response of human aortic valve interstitial cells via modulation of ERK1/2 and nuclear factor- $\kappa \mathrm{B}$ activation. Arterioscler Thromb Vasc Biol 2013;33:1580-90.

[28] Durbin AD, Gotlieb AI. Advances towards understanding heart valve response to injury. Cardiovasc Pathol 2002;11:69-77.

[29] Towler DA. Molecular and cellular aspects of calcific aortic valve disease. Circ Res 2013;113:198208.

[30] Lam NT, Balachandran K. The Mechanobiology of Drug-Induced Cardiac Valve Disease. J LongTerm Eff Med 2015;25.

[31] Görnemann T, Hübner H, Gmeiner P, Horowski R, Latté KP, Flieger M, et al. Characterization of the molecular fragment that is responsible for agonism of pergolide at serotonin 5-hydroxytryptamine2B and 5-hydroxytryptamine2A receptors. J Pharmacol Exp Ther 2008;324:1136-45.

[32] Patel C, Mathur M, Escarcega RO, Bove AA. Carcinoid heart disease: Current understanding and future directions. Am Heart J 2014;167:789-95.

[33] Pritchett AM, Morrison JF, Edwards WD, Schaff HV, Connolly HM, Espinosa RE. Valvular heart disease in patients taking pergolide. Mayo Clinic Proceedings: Elsevier; 2002 p. 1280-6.

[34] Zadikoff C, Rochon P, Lang A. Cardiac valvulopathy associated with pergolide use. Can J Neurol Sci 2006;33:27-33.

[35] Calomne V, Dupuis M, Muller T, Kempinere F. [Reversible cardiac valve abnormalities with pergolide treatment]. Rev Neurol 2004;160:81-2.

[36] Yip CYY, Simmons CA. The aortic valve microenvironment and its role in calcific aortic valve disease. Cardiovasc Pathol 2011;20:177-82.

[37] Jana S, Lerman A, Simari RD. An In Vitro Model of a Fibrosa Layer of a Heart Valve. ACS Appl Mater Interfaces 2015.

[38] Grosberg A, Nesmith AP, Goss JA, Brigham MD, McCain ML, Parker KK. Muscle on a chip: in vitro contractility assays for smooth and striated muscle. J Pharmacol Toxicol 2012;65:126-35.

[39] Grosberg A, Alford PW, McCain ML, Parker KK. Ensembles of engineered cardiac tissues for physiological and pharmacological study: heart on a chip. Lab Chip 2011;11:4165-73.

[40] Agarwal A, Goss JA, Cho A, McCain ML, Parker KK. Microfluidic heart on a chip for higher throughput pharmacological studies. Lab Chip 2013;13:3599-608.

[41] Alford PW, Feinberg AW, Sheehy SP, Parker KK. Biohybrid thin films for measuring contractility in engineered cardiovascular muscle. Biomaterials 2010;31:3613-21.

[42] Balachandran K, Alford PW, Wylie-Sears J, Goss JA, Grosberg A, Bischoff J, et al. Cyclic strain induces dual-mode endothelial-mesenchymal transformation of the cardiac valve. PNAS 2011;108:199438.

[43] Badrossamay MR, McIlwee HA, Goss JA, Parker KK. Nanofiber assembly by rotary jet-spinning. Nano Lett 2010;10:2257-61.

[44] Mellado P, McIlwee HA, Badrossamay MR, Goss JA, Mahadevan L, Parker KK. A simple model for nanofiber formation by rotary jet-spinning. Appl Phys Lett 2011;99:203107.

[45] Badrossamay MR, Balachandran K, Capulli AK, Golecki HM, Agarwal A, Goss JA, et al. Engineering hybrid polymer-protein super-aligned nanofibers via rotary jet spinning. Biomaterials 2014;35:3188-97. 
[46] Golecki HM, Yuan H, Glavin C, Potter B, Badrossamay MR, Goss JA, et al. Effect of Solvent Evaporation on Fiber Morphology in Rotary Jet Spinning. Langmuir 2014;30:13369-74.

[47] Hoerstrup SP, Sodian R, Daebritz S, Wang J, Bacha EA, Martin DP, et al. Functional living trileaflet heart valves grown in vitro. Circulation 2000;102:Iii-44-Iii-9.

[48] Schmidt D, Dijkman PE, Driessen-Mol A, Stenger R, Mariani C, Puolakka A, et al. Minimallyinvasive implantation of living tissue engineered heart valves: a comprehensive approach from autologous vascular cells to stem cells. J Am Coll Cardiol 2010;56:510-20.

[49] Driessen-Mol A, Emmert MY, Dijkman PE, Frese L, Sanders B, Weber B, et al. Transcatheter implantation of homologous "off-the-shelf" tissue-engineered heart valves with self-repair capacity: longterm functionality and rapid in vivo remodeling in sheep. J Am Coll Cardiol 2014;63:1320-9.

[50] Huang H-S, Huang S. Real-time strain mapping via biaxial stretching in heart valve tissues. Engineering in Medicine and Biology Society (EMBC), 2012 Ann Intern Conf IEEE: IEEE; 2012. p. 6653-6.

[51] Setola V, Hufeisen SJ, Grande-Allen KJ, Vesely I, Glennon RA, Blough B, et al. 3, 4Methylenedioxymethamphetamine (MDMA, "Ecstasy") induces fenfluramine-like proliferative actions on human cardiac valvular interstitial cells in vitro. Mol Pharmacol 2003;63:1223-9.

[52] Jähnichen S, Horowski R, Pertz HH. Agonism at 5-HT 2B receptors is not a class effect of the ergolines. Eur J Pharmacol 2005;513:225-8.

[53] Bray MA, Sheehy SP, Parker KK. Sarcomere alignment is regulated by myocyte shape. Cell Motil Cytoskel 2008;65:641-51.

[54] Grosberg A, Kuo P-L, Guo C-L, Geisse NA, Bray M-A, Adams WJ, et al. Self-organization of muscle cell structure and function. PLoS Comput Biol 2011;7:e1001088.

[55] Xu S, Liu AC, Kim H, Gotlieb AI. Cell density regulates in vitro activation of heart valve interstitial cells. Cardiovasc Pathol 2012;21:65-73.

[56] Baseman D, O'suilleabhain P, Reimold S, Laskar S, Baseman J, Dewey R. Pergolide use in Parkinson disease is associated with cardiac valve regurgitation. Neurology 2004;63:301-4.

[57] Sherlock M, Toogood AA, Steeds R. Dopamine agonist therapy for hyperprolactinaemia and cardiac valve dysfunction; a lot done but much more to do. Heart 2009;95:522-3.

[58] Go OD, Safar ME, Smulyan H. Assessment of aortic stiffness by transesophageal echocardiography. Echocardiography 2014;31:1105-12.

[59] Wentland AL, Grist TM, Wieben O. Review of MRI-based measurements of pulse wave velocity: a biomarker of arterial stiffness. Cardiovasc Diagn Ther 2014;4:193.

[60] Schoen FJ. Cardiac valves and valvular pathology: update on function, disease, repair, and replacement. Cardiovasc Pathol 2005;14:189-94.

[61] Hinz B. The myofibroblast: paradigm for a mechanically active cell. J Biomech. 2010;43:146-55.

[62] Bravo-Cordero JJ, Magalhaes MA, Eddy RJ, Hodgson L, Condeelis J. Functions of cofilin in cell locomotion and invasion. Nat Rev Mol Cell Biol 2013;14:405-15.

[63] Hinz B, Phan SH, Thannickal VJ, Galli A, Bochaton-Piallat M-L, Gabbiani G. The myofibroblast: one function, multiple origins. Am J Pathol 2007;170:1807-16.

[64] Pho M, Lee W, Watt DR, Laschinger C, Simmons CA, McCulloch C. Cofilin is a marker of myofibroblast differentiation in cells from porcine aortic cardiac valves. Am J Physiol-Heart C 2008;294:H1767-H78.

[65] Zhao X-H, Laschinger C, Arora P, Szászi K, Kapus A, McCulloch CA. Force activates smooth muscle $\alpha$-actin promoter activity through the Rho signaling pathway. J Cell Sci 2007;120:1801-9.

[66] Shim J, Grosberg A, Nawroth JC, Parker KK, Bertoldi K. Modeling of cardiac muscle thin films: pre-stretch, passive and active behavior. J Biomech 2012;45:832-41.

[67] Nesmith AP, Agarwal A, McCain ML, Parker KK. Human airway musculature on a chip: an in vitro model of allergic asthmatic bronchoconstriction and bronchodilation. Lab Chip 2014;14:3925-36.

[68] Capulli A, Tian K, Mehandru N, Bukhta A, Choudhury S, Suchyta M, et al. Approaching the in vitro clinical trial: engineering organs on chips. Lab Chip 2014;14:3181-6. 
[69] Huh D, Torisawa Y-s, Hamilton GA, Kim HJ, Ingber DE. Microengineered physiological biomimicry: organs-on-chips. Lab Chip 2012;12:2156-64.

[70] Balachandran K, Hussain S, Yap C-H, Padala M, Chester AH, Yoganathan AP. Elevated cyclic stretch and serotonin result in altered aortic valve remodeling via a mechanosensitive 5-HT 2A receptordependent pathway. Cardiovasc Pathol 2012;21:206-13.

[71] Elangbam CS. Drug-induced valvulopathy: an update. Toxicol Pathol 2010;38:837-48.

[72] Gardin JM, Schumacher D, Constantine G, Davis KD, Leung C, Reid CL. Valvular abnormalities and cardiovascular status following exposure to dexfenfluramine or phentermine/fenfluramine. Jama 2000;283:1703-9.

[73] Yamamoto M, Uesugi T, Nakayama T. Dopamine agonists and cardiac valvulopathy in Parkinson disease A case-control study. Neurology 2006;67:1225-9.

[74] Weston MW, Yoganathan AP. Biosynthetic activity in heart valve leaflets in response to in vitro flow environments. Ann Biomed Eng 2001;29:752-63.

[75] Simmons CA. Aortic valve mechanics: an emerging role for the endothelium. J Am Coll Cardiol 2009;53:1456-8. 